\title{
Clinical Outcomes in High-Risk Pregnancies Due to Advanced Maternal Age
}

\author{
Rosaly Correa-de-Araujo, MD, MSc, PhD, and Sung Sug (Sarah) Yoon, RN, PhD²
}

\begin{abstract}
Although the influence of advanced maternal age (AMA) and delayed childbearing on adverse maternal and perinatal outcomes has been studied extensively, no universal consensus on the definition of AMA exists. This terminology currently refers to the later years of a woman's reproductive life span and generally applies to women age $\geq 35$ years. AMA increases the risk of pregnancy complications, including ectopic pregnancy, spontaneous abortion, fetal chromosomal abnormalities, congenital anomalies, placenta previa and abruption, gestational diabetes, preeclampsia, and cesarean delivery. Such complications could be the cause of preterm birth and increase the risk of perinatal mortality. For women who have a chronic illness, pregnancy may lead to additional risk that demands increased monitoring or surveillance. The management of pregnant women of AMA requires understanding the relationship between age and preexisting comorbidities. The outcomes from pregnancy in AMA may have a negative impact on women's health as they age because of both the changes from the pregnancy itself and the increased risk of pregnancy-related complications. Postpartum depression affects women of AMA at higher rates. Links between preeclampsia and the risk of future development of cardiovascular disease require follow-up surveillance. The association between hypertensive pregnancy disorders and cognitive and brain functions needs further investigation of sex-specific risk factors across the life span. Educating providers and women of AMA is crucial to facilitate clinical decision making and such education should consider cultural influences, risk perception, and women's health literacy, as well as providers' biases and system issues.
\end{abstract}

Keywords: advanced maternal age, pregnancy outcomes, adverse maternal outcomes, maternal mortality and morbidity, beliefs and behaviors, health equity

\section{Introduction}

A TREND HAS developed worldwide for women to delay childbearing into their 30s and, in some cases, their 40s. ${ }^{1,2}$ According to the Centers for Disease Control and Prevention, the number of pregnancies in women of advanced maternal age (AMA) continues to escalate in the United States, especially among women $\geq 40$ years. In 2014, $9 \%$ of first births were to women age $\geq 35$ years, an increase of $23 \%$ from $2000 .^{3}$ Numerous reasons underlie the increased rates of AMA pregnancies or births. ${ }^{4-7}$ Demographic data show an increased population of women age 35-45 influenced by evolving social and cultural changes, including higher rates of divorce, having multiple partners before settling down, living together before marriage, and having a later or second marriage. Women with higher socioeconomic status (SES) and higher level of education tend to delay motherhood into their mid-to-late 30s. Advances in medical sciences have provided women with better contraceptive options and more available fertility treatment, but SES affects access to and utilization of assisted reproductive technology (ART). Limited job-related policies (e.g., unavailability or

\footnotetext{
${ }^{1}$ Division of Geriatrics and Clinical Gerontology, U.S. Department of Health and Human Services, National Institute on Aging, National Institutes of Health, Bethesda, Maryland, USA.

${ }^{2}$ Division of Extramural Science Programs, U.S. Department of Health and Human Services, National Institute of Nursing Research, National Institutes of Health, Bethesda, Maryland, USA.

(C) Rosaly Correa-de-Araujo et al. 2021; Published by Mary Ann Liebert, Inc. This Open Access article is distributed under the terms of the Creative Commons Attribution Noncommercial License (http://creativecommons.org/licenses/by-nc/4.0/) which permits any noncommercial use, distribution, and reproduction in any medium, provided the original author(s) and the source are cited.
}

Correction added on February 19, 2021 after first online publication of November 13, 2020: The article reflects Open Access, with copyright transferring to the author(s), and a Creative Commons Attribution Noncommercial License (CC-BY-NC) added (http:// creativecommons.org/licenses/by-nc/4.0/). 
limitations with childcare, low benefit levels, policies unfavorable to motherhood and work/career) are among other reasons for delaying motherhood. Education and job opportunities thus contribute to delaying motherhood to later in life. Maternal education is shown to be among the strongest predictors of contraceptive use, with college-educated women generally having low first-birth rates in their 20 s and higher birth rates in their 30s.

Despite having become more common, older expectant mothers remain a stigmatized social identity, ${ }^{8}$ with some still referring to the outdated term "geriatric pregnancy." Although the impact of AMA and delayed childbearing on maternal and perinatal outcomes has been studied extensively, no universal consensus on its definition exists. This terminology currently refers to the later years of a woman's reproductive life span and generally applies to women age $\geq 35$ years. ${ }^{9,10}$ The effects of increasing age, however, occur as a continuum, with a decline in fertility, particularly observed after their mid-30s. Regardless of the age used to define AMA, pregnancies in women age $\geq 35$ years are considered at risk of both obstetric complications and interventions.

\section{Culture and Risk Perception of Providers and Women}

Comparing studies assessing pregnancy risk perception of providers, midwives, and women is challenging because of the differences in their methodological approaches. ${ }^{11}$ Risk perception is multifaceted and influenced by various personal, psychological, and societal factors. Differences in perception among providers, midwives, and women have been attributed to women's more subjective views and limited knowledge related to risk. Most pregnant women express fears with the birth process and the wellbeing of babies, but the risk usually is accepted as part of the psychological strategies of pregnancy that women use to cope with their apprehensions. These strategies likely help them endure the higher degrees of risk, because they believe that better outcomes for themselves and for their babies will be reached. ${ }^{11}$ Women generally go beyond the medical and social models of care to include feelings of experienced risk and resilience factors (e.g., prior use of ART). ${ }^{8,11}$ Providers express a more objective and science-based perception because they focus on the biomedical risk and physiological outcomes. Midwives adopt a more holistic attitude that encompasses the psychological and social wellbeing of the patient.

Culture contributes to increased maternal mortality because of harms to the mother during the perinatal period (e.g., direct harmful acts, inaction, use of care, and social status). Direct harmful practices and factors that contribute to maternal mortality include burning, cutting, food and water shortages, forced or delayed placental expulsion, and exposure to infectious agents. A woman may not respond adequately to prevent adverse outcomes; obstetric emergencies may not be recognized, or cultural beliefs about their causes, treatment, and implications may preclude her from seeking help. Culture may affect maternal mortality because of a lack of or limited access to health services during pregnancy and childbirth. ${ }^{12,13}$ Several factors may contribute to a woman's perception of pregnancy risk, including medical risk, psychological factors, risk characteristics, stage of pregnancy, and the judgment of health care providers. ${ }^{14}$

\section{Preexisting Comorbidities and Impact on Pregnancy Outcomes}

For women living with a chronic illness, pregnancy may be experienced as a time of additional risk that demands increased monitoring or surveillance. ${ }^{15}$ The management of pregnant women of AMA requires understanding the interplay between age and preexisting comorbidities. ${ }^{16,17}$ The aging process alone contributes to some obstetric complications, and other complications largely relate to additional coexisting factors or conditions (e.g., multiple gestation, higher parity, chronic diseases). With aging, the prevalence of coexisting conditions (e.g., surgical illnesses, cancer, diabetes, obesity, and cardiovascular, renal, and autoimmune diseases) increases considerably, putting pregnant women age $\geq 35$ years at high risk of experiencing two- to three-fold higher rates of hospitalization, cesarean delivery, and other pregnancy-related complications. ${ }^{17-20}$

Primiparas of AMA are more likely to be single, have chronic conditions, and have higher rates of gestational diabetes, gestational hypertension, and preeclampsia/eclampsia. Multiple pregnancies hold additional risk and may reduce the effect of age. In multivariable analysis, being of age $\geq 45$ was an independent risk factor for gestational diabetes, gestational hypertension, and preeclampsia/eclampsia.

Preexisting and pregnancy-related hypertension are the most common complicating conditions, particularly because of their high prevalence in older women and in those who are overweight. The chances of being diagnosed with chronic hypertension are two- to four-fold higher in women age $\geq 35$ years (vs. 30-34 years). ${ }^{18}$ Hypertensive pregnancy disorders (HPDs)_including gestational hypertension, preeclampsia/eclampsia, chronic hypertension, and preeclampsia or eclampsia superimposed to chronic hypertension-are major causes of maternal and fetal morbidity and mortality that affect $\sim 12 \%$ of all pregnancies.

Preexisting diabetes and gestational diabetes increase threeto six-fold in women age $\geq 40$ years (vs. 20-29 years). ${ }^{19,21-24}$ Preexisting diabetes is associated with increased risks of congenital anomalies and perinatal mortality and morbidity. The incidence of gestational diabetes in the general obstetric population is $3 \%$, increasing to $7 \%-12 \%$ in women age $>40$ years and to $20 \%$ in those age $>50$ years. ${ }^{19,23,24}$ A major complication of gestational diabetes is macrosomia. ${ }^{25}$

\section{Pregnancy Outcomes in AMA}

Maternal mortality rate (MMR) has increased considerably in the United States during the past 25 years and is currently the highest among developed countries. In 2016, 658 women died of maternal causes, with a MMR of 17.4 deaths per 100,000 live births; racial and ethnic groups were affected disproportionately, with the MMR for non-Hispanic Black women (37.1 deaths/100,000 live births) being 2.5 times the rate for non-Hispanic White women (14.7) and 3.1 times the rate for Hispanic women (11.8). ${ }^{26}$ Age, disabilities, geographic areas, and social and structural determinants of health are significant contributing factors. One-third of pregnancy-related deaths occur during pregnancy, one-third during or in the week after delivery, and one-third between 1 week and 1 year postpartum. The leading mortality causes in the United States include hemorrhage, infection, and cardiovascular disease. 
Although all women can suffer complications early or later in pregnancy, the risk for some of these complications is higher with AMA. From 1991 to 1997, the risk of pregnancyrelated mortality in the United States was five-fold higher for women age $>40$ years and more than double for women age 35-39 years compared with that of women age 25-29 (9 vs. 21 vs. 46/100,000 live births, respectively). ${ }^{27,28}$ In 2016, the MMR in the United States increased with successively older age groups, with the rate for women age $\geq 40$ years (81.9) equal to 7.7 times that for women age $<25$ years $(10.6) .{ }^{26}$ In developing countries, maternal mortality remains a significant problem; limitations in access to and quality of care contribute significantly to maternal losses, but maternal characteristics (e.g., increasing age, parity) also are important factors. ${ }^{29}$

\section{Early pregnancy outcomes}

Ectopic pregnancy. Maternal age of $\geq 35$ years is associated with a four- to eight-fold increased risk of ectopic pregnancy, a major cause of maternal mortality and morbidity in early pregnancy. ${ }^{30,31}$ This finding likely reflects accumulated risk factors over time (e.g., multiple sexual partners, pelvic infection, tubal conditions).

Gene and chromosomal abnormalities, congenital malformations. Except for ART, the data on AMA's effect on gene abnormalities (e.g., single gene disorders, epigenetic events) are limited. Epidemiological studies show an association between advanced maternal and paternal ages and the risk of autism spectrum disorders in the offspring. ${ }^{32,33}$ As women age, a stable increase in the risk of chromosomal abnormalities (e.g., aneuploidy [autosomal trisomy]) is reported in karyotype analysis from spontaneous abortions, pregnancy terminations, genetic amniocenteses, and both live-born and stillborn infants. ${ }^{34,35}$ These age-related errors seem to augment the risk of nondisjunction resulting in unequal chromosome products at completion of cell division. Furthermore, the risk of AMA-associated nonchromosomal anomalies includes the highest rates of cardiac anomalies. ${ }^{36}$ AMA also is a risk factor for Down's syndrome; mothers who give birth to a child with this syndrome often have characteristics consistent with accelerated aging. ${ }^{37,38}$

Spontaneous abortion. With AMA, the rates of spontaneous abortion increase, with most losses occurring between 6 and 14 weeks of pregnancy. ${ }^{39,40}$ Trisomic and euploid losses are linked to a decline in the quality of the oocyte and possible functional changes (i.e., uterine, hormonal). An overall rate of $11 \%$ in spontaneous abortion has been reported in a large Scandinavian study of hospitalized women. ${ }^{30}$ The risk of loss in different age groups was based on the hypothesis that only $80 \%$ of women with abortions were hospitalized. Higher rates of loss were associated with AMA versus younger age groups ( $<30$ years, $12 \% ; 30-34$ years, $15 \%$; 35-39 years, $25 \%$; $40-44$ years, $51 \%$; $\geq 45$ years, $93 \%$ ). Spontaneous abortion was affected by maternal age independently of parity and previous abortion history. However, Magnus et al. ${ }^{40}$ found that the risk of miscarriage varied significantly with maternal age, showed a strong pattern of recurrence, and was increased after adverse pregnancy outcomes. This risk increased if there was a history of prior preterm delivery, stillbirth, cesarean section, or gestational diabetes. Women who were small for their baby's gestational age were at slightly greater risk for miscarriage. These findings suggest that biological conditions or unmeasured common risk factors may be among the shared underlying causes for miscarriages and other complications. The highest rates of spontaneous abortion with AMA have been confirmed in a study of pregnancies resulting from ART use. ${ }^{41,42}$

Multiple gestation. An increased prevalence of multiple gestation is seen with AMA. Multiple gestation is related to a higher risk of naturally conceived twins and higher ART use. In contrast to singletons, the outcome of multiple pregnancies in AMA is as good or better than the outcome reported for younger women. ${ }^{43}$

\section{Late pregnancy outcomes}

Preeclampsia. Preeclampsia affects $4 \%-8 \%$ of all pregnancies. The rates of preeclampsia have increased by $25 \%$ over the past two decades. ${ }^{44}$ In the general obstetric population, the incidence of preeclampsia is $3 \%-4 \%$, increasing to $5 \%-10 \%$ in women $>40$ years of age, becoming as high as $35 \%$ in those $>50$ years. $^{23,24}$ In a large study in Ontario (2012-2015) mothers at very AMA were at greater risk of developing multiple adverse outcomes consisting of preeclampsia, intrauterine growth retardation, stillbirth, or placental abruption. ${ }^{45}$

Interpregnancy interval. Maternal and perinatal outcomes at different birth intervals in a cohort of 148,000 pregnancies revealed an increased risk of severe maternal morbidity or mortality for women $\geq 35$ years of age at the time of index birth. Women at 6-month interpregnancy intervals presented with adverse fetal and neonatal outcomes, particularly spontaneous preterm delivery. Women age 20-34 years giving birth at a similar time interval showed no such risks. ${ }^{21}$

Placenta previa and placental abruption. AMA is linked to a higher prevalence of placenta previa and placental abruption, both of which cause bleeding in late pregnancy (after about 20 weeks). Placental abruption is a rare but serious complication in the second half of pregnancy and lifethreatening for both the mother and the fetus. Death is inevitable if a complete or near-placental separation occurs, unless an immediate cesarean section is performed. Fetal mortality rates of $1 \%-40 \%$ have been reported, but these rates depend on the age of the fetus and extent of separation. Around $1 \%-5 \%$ of maternal deaths each year are linked to placental abruption. ${ }^{46}$ Mothers at very AMA also are at a higher risk for placental abruption. ${ }^{45}$ Multiparity is associated with a considerable proportion of the excess risk for both placental abruption and placenta previa; however, no significant correlation was observed between maternal age and abruption when parity and hypertension were considered.

Age and parity seem to be independent risk factors for placenta previa. Despite a small absolute risk $(0.25 \%$ vs. $0.03 \%$ ), nulliparous women age $\geq 40$ years have a 10 -fold increased risk of placenta previa compared with nulliparous women age $20-29$ years. $^{21}$

Amniotic fluid embolism and obstetric shock. Although the etiology of amniotic fluid embolism remains unknown, the embolism may occur in healthy women during labor, including cesarean section; after abnormal vaginal delivery; 
during the second trimester of pregnancy; up to 48 hours postdelivery or during abortion; after abdominal trauma; and during amnioinfusion. ${ }^{47}$ In a population-based study (830,000 singleton births), women $\geq 40$ years of age showed an eight-fold increased risk of amniotic fluid embolism and a three-fold increased risk of obstetric shock compared with women age $25-29$ years. ${ }^{48}$

Peripartum cardiomyopathy. Peripartum cardiomyopathy is a rare, generally dilated form of cardiomyopathy with systolic dysfunction that presents in late pregnancy or, more commonly, in the early postpartum period. Black-descendent women are at highest risk, with high rates seen in Nigeria and Haiti. Preeclampsia, AMA, and multiple gestation pregnancy are among the risk factors for cardiomyopathy. Although its pathophysiology is still unclear, vascular/hormonal pathways likely play a role in women with underlying susceptibility (e.g., sarcomere gene mutation). In general, more than half of affected women recover from systolic function, but some develop chronic cardiomyopathy, and few require mechanical support or cardiac transplantation (or both). Thromboembolism and arrhythmias are potential negative outcomes, as well. ${ }^{49}$

Cesarean delivery. The optimal gestational age for labor or cesarean delivery at AMA is unclear. Some support delivery in the 39th week of gestation. ${ }^{50}$ Studies consistently report that women $\geq 35$ years of age are more likely to experience labor dystocia ${ }^{51}$ and undergo a cesarean procedure. $^{14,21,22,24,52} \mathrm{~A}$ cohort of $>78,000$ singleton births in the United States (2003-2012) showed that the proportion of a primary cesarean delivery (PCD) increased with age for both primiparous and multiparous women. ${ }^{14}$ The PCD rate was $20 \%$ for women age $25-34$ years, $26 \%$ for those $35-39$ years, $31 \%$ for those $40-44$ years, $36 \%$ for those $45-49$ years, and $61 \%$ for those $\geq 50$ years. The overall PCD rate for singleton births in the United States was $\sim 22 \%$ during a similar time period. ${ }^{53}$ The reasons for such high rates in older women are controversial, but they involve an increased frequency of medical complications, labor induction, fetal malposition, and a lower threshold among patients and physicians for performing a cesarean delivery. Maternal request for cesarean delivery is becoming more common, particularly among women of AMA. ${ }^{54}$ These women appear to have an increased risk of failure of labor to progress normally.

Throughout the childbearing years, the association between uterine dysfunction increases with maternal age.$^{55-57}$ Findings have not been consistent in recent studies that examine the effect of age on the length of the first stage of labor; however, the length of the second stage seems to increase as age increases. ${ }^{57,58}$ Despite the impact of age on uterine function, a meta-analysis of five trials $(>2,600$ women) reported that labor induction at term in women age $\geq 35$ years did not increase the cesarean delivery rate compared with women undergoing expectant management. ${ }^{59}$ Women of AMA subjected to a trial of labor after a previous cesarean delivery appear to be at increased risk for both failed trial and uterine rupture. ${ }^{60,61}$ Regardless of the known risks, older pregnant women frequently are treated as "higher risk," resulting in elevated rates of cesarean section for nonmedical reasons and more frequent induced labor. ${ }^{62-65}$ Because worse outcomes are more prevalent in this group, poorer outcomes are likely to increase with increasing age. ${ }^{65}$
Preterm delivery, low birth weight, and stillbirth. Few studies have examined the associations between maternal age and pregnancy perinatal outcomes in low- and middleincome countries. Data from Consortium for Health Orientated Research in Transitioning Societies (COHORTS), a collaboration of five birth cohorts from low- and middleincome countries (Brazil, Guatemala, India, the Philippines, and South Africa) evaluated mothers who were recruited before or during pregnancy, and their children were followed up to adulthood. ${ }^{66}$ Unadjusted and adjusted analyses for maternal SES, height, parity, and breastfeeding duration were conducted using data from 22,188 mothers (1969-1989). After adjustment, AMA remained associated with increased risk of preterm birth, but children of older mothers had less 2year stunting and failure to complete secondary schooling compared with those whose mothers were age 20-24 years. Extreme maternal ages showed higher adult fasting glucose concentrations (roughly $0.05 \mathrm{mmol} / \mathrm{L}$ ), likely reflecting compromised offspring glucose metabolism.

Several population-based studies have documented the link between AMA and increased rates of low birth weight (LBW) and preterm delivery. ${ }^{1,19,23,67-69}$ A cohort of 173,715 healthy nulliparous women delivering singletons showed that being of age 35-40 related to a significantly higher risk of very and moderate LBW and preterm birth and to a smallfor-gestational-age infant compared birth outcome. ${ }^{1}$ A subsequent study $(32,000$ women $\geq 40$ years $)$ confirmed the increased risk of preterm delivery after adjusting for confounders (e.g., multiple gestation, smoking, parity, maternal disease). ${ }^{70}$ The rates of preterm delivery in $<32$ weeks for women age 20-29 years, 40-44 years, and $\geq 45$ years were $1.01 \%, 1.80 \%$, and $2.24 \%$, respectively. In the United States, the adjusted odds ratios (OR) for delivering an LBW White infant increased progressively, with each 5-year increase in maternal age, reaching 2.3 in women $\geq 40$ years of age. ${ }^{68}$ The maternal age effect on both very LBW and preterm delivery was similar. Although women of AMA have more preterm deliveries, their neonates are not at an increased risk for morbidity compared with those of younger women.

Large studies worldwide consistently report a significant increased risk for stillbirth in women age $\geq 35$ years. A systematic review and meta-analysis showed that the maternal age of $>35$ years was associated with a $65 \%$ increased risk of a stillbirth, with a higher relative risk at age 40 years. ${ }^{71}$ This risk is most notable after $\sim 37$ weeks of gestation. ${ }^{72,73}$ In addition, the extra perinatal mortality involving women of AMA is mainly caused by nonanomalous fetal deaths (often unexplained), even after adjusting risk factors (e.g., hypertension, diabetes, antepartum bleeding, smoking, multiple gestation). ${ }^{9,70,73-76}$ In developed countries, the absolute risk of stillbirth is small, even at very AMA. In a Swedish study, the absolute risk of an intrauterine fetal death at $\geq 28$ weeks of gestation or death of the live-born child within the first 28 days of life was $1.1 \%$ in women age $\geq 40$ years (343 deaths/31,662 deliveries) and $1.7 \%$ in women age $\geq 45$ years ( 20 deaths $/ 1,205$ deliveries) after adjusting for confounders (e.g., parity, congenital malformations, smoking, maternal disease) versus women age $20-29$ years $\left(5,246\right.$ deaths $/ 876,361$ deliveries). ${ }^{70}$ In the United States, the risk of stillbirth at 37-41 weeks for primiparous women increased considerably with maternal age in an analysis of more than 5 million nonanomalous singleton gestations $^{73}: 3.73$ (women $<35$ years), 6.41 (35-39 years), and 
8.65 ( $>40$ years) per 1,000 ongoing pregnancies. This persisted after controlling for disease and race/ethnicity and increased abruptly at 40 weeks of gestation, suggesting that women of AMA are "post term" sooner than younger women.

Antepartum and postpartum hemorrhage. An adjusted analysis of a cohort (37 million deliveries, 2006-2015) demonstrated that women age $45-54$ years had 3.5 times the risk of severe maternal morbidity and showed the highest rates of cesarean delivery, preeclampsia, postpartum hemorrhage, gestational diabetes, thrombosis, and hysterectomy. ${ }^{77}$ Another cohort study $\left(64,886\right.$ pregnant women $\left.{ }^{78}\right)$ also analyzed multiple risk factors and found that women age $\geq 35$ years $(n=12,686)$ had increased frequency for antepartum hemorrhage, placenta previa, hypertension, gestational diabetes, and overweight or obesity. In the multiple logistic regression analysis, advanced age had a negative effect. Authors concluded that AMA served as a surrogate factor for postpartum hemorrhage due to the associated increased risk factors, obstetric complications, and interventions.

\section{Subsequent Health Postpregnancy in AMA}

Outcomes from pregnancy in AMA may negatively affect health as women continue to age, because of both pregnancyinduced changes and the increased risk of pregnancy-related complications. The findings from the Women's Health Initiative show a trend toward increased risk of hemorrhagic stroke in women who had their last pregnancy at age $\geq 40$ years versus before age $40 .^{79}$ Preeclampsia, a risk factor for future cardiovascular disease, is also much more common in women of AMA. ${ }^{79}$ Israeli women giving birth at age $\geq 45$ years had about $40 \%$ lower mortality risk compared with women who gave birth before age $35 .^{80}$ Further studies are needed to investigate mortality risk in AMA, because factors other than reproductive age may be involved, such as early life stressors and reproduction accelerating aging. Women who have both will likely be most affected. ${ }^{81}$

Postpartum depression is a relatively common, potentially debilitating condition, but its relationship with AMA has not been studied adequately. Women of AMA, however, have significantly higher rates of depression. The prevalence of depression among women who delivered recently was significantly higher in those age 40-44 years versus 30-35 years (adjusted OR $3.72 ; 95 \%$ confidence interval 2.15-6.41). ${ }^{82}$ Research is required to determine whether a targeted screening and prevention program can help reduce the burden of depression among older mothers.

Because evidence supports the link between preeclampsia and the risk of future cardiovascular disease in affected pregnancies, appropriate preconception, prenatal, and postpartum education is needed, as well as surveillance, to improve the long-term health of both the mother and the infant. No evidencebased preventive interventions are available for cardiovascular disease for women who suffer preeclampsia and their children. However, these women may require yearly risk assessments and education (e.g., smoking cessation, increasing physical activity, maintaining a healthy diet and weight). Preeclampsia should be acknowledged by providers as a cardiovascular disease risk factor, followed by implementation of appropriate monitoring, education, and cardiovascular preventive strategies. Studies show on associations between HPDs and subjective cognitive complaints or brain white matter hyperintensities observed in magnetic resonance imaging. The long-term association between HPDs (i.e., >10 years after pregnancy) and brain structure and cognitive function ${ }^{83}$ indicates that women with a history of HPDs are more likely to have brain atrophy decades after their pregnancies compared with women with normotensive pregnancies. More white matter hyperintensities are seen in those with HPDs. Given the greater prevalence of HPDs with increasing obesity and AMA, research examining HPDs and the risk of Alzheimer's disease is warranted. Whether a common underlying factor exists that augments the risk of both HPDs and Alzheimer's disease or whether HPDs are an independent risk factor requires further clarification. ${ }^{84}$ Gaining a better understanding of sex-specific risk factors across the life span will contribute to developing and implementing specific preventive strategies and therapeutic interventions for women at risk for future occurrence of dementias as part of a subsequent impact of pregnancy later in life.

Research on centenarians shows that women who had their last child after age 33 had twice the odds of living to age 95 years compared with women who had their last child by the age of $\leq 30$ years. $^{85}$ Interpretation on how biological, socioeconomic, educational, and environmental influences interact to support these findings is conflicting, and their interaction has been proposed as part of the complexity of the age-based reproductive health care stereotype threat concept, opening multiple research opportunities. ${ }^{8}$

\section{Conclusion: Empowering Providers and Women of AMA}

Empowering providers and women of AMA is critical to facilitating clinical decision making. Education on AMAassociated risks will help women make more informed decisions on the timing of childbearing and are essential to patient-centered care. Information should be delivered in a culturally competent way that also takes health literacy into account. This means that providers should minimize or eliminate their biases and be educated about the influences of religion, culture, beliefs, and behaviors, including pregnancy risk perception by women of AMA. Communication with women with varying backgrounds should rely on the sensitive and respectful assessment of their understanding and perception of risk and safety and should acknowledge any anxieties they may have. Providers should also be aware of the subjectivity of their own professional team and risk assessments and factors that may influence their perceptions. ${ }^{11}$

Further research is required to examine the most perceived pregnancy and birth-related risk, the causes of those perceived concerns, and the extent to which evidence supports these perceptions. How the provider's perception of risk influences communication with pregnant women, creates barriers to effective risk communication, and the ways that this communication can be improved should be investigated. Research into professional interactions should explore how stereotypical expectations about the risk perceptions of other groups may arise and can be challenged to improve the communication between disciplines. ${ }^{11}$ Thoughtful messages of age diversity in health care environments and other maternal care facilities can reduce negative perceptions of age-related reproductive health stereotypes and any potential for psychosocial, affective, 
behavioral, cognitive, psychophysiological, and relational harms. Similarly, positive public health information regarding the health of older expectant mothers and their children can help reduce age-based reproductive health care stereotyping. Additional research also should focus on longitudinal studies to clarify the role of age stereotyping in health care and disparities among women of reproductive age. ${ }^{8}$

\section{Disclaimer}

Any opinions or recommendations expressed in this publication are those of the authors and do not necessarily reflect the views of the National Institute on Aging, the National Institute of Nursing Research, National Institutes of Health, or the U.S. Department of Health and Human Services.

\section{Author Disclosure Statement}

R.C.-d.A. conceptualized and drafted the first version of the article. Both authors reviewed, discussed, and finalized the content of the article. No competing financial interests exist.

\section{Funding Information}

No funding was received for this article.

\section{References}

1. Tough SC, Newburn-Cook C, Johnston DW, et al. Delayed childbearing and its impact on population rate changes in lower birth weight, multiple birth, and preterm delivery. Pediatrics 2002;109:399-403.

2. Chan BCP, Lao TT. Effect of parity and advanced maternal age on obstetric outcome. Obstet Gynecol Surv 2008;63: 761-763.

3. Martin JA, Hamilton BE, Osterman MJK, et al. Births: Final data for 2016. National vital statistics reports; vol. 67, no. 1. Hyattsville, MD: National Center for Health Statistics, 2018.

4. Cook A, Mills TA, Lavender T. Informed and uninformed decision making-women's reasoning, experiences, and perceptions with regard to advanced maternal age and delayed childbearing: A meta-synthesis. Int J Nurs Stud 2010; 47:1317-1329.

5. Mills M, Rindfuss RR, McDonald P, et al. ESHRE reproduction and society task force. Why do people postpone parenthood? Reasons and social policy incentives. Hum Reprod Update 2011;17:848-860.

6. Chambers GM, Hoang VP, Illingworth PJ. Socioeconomic disparities in access to ART treatment and the differential impact of a policy that increased consumer costs. Hum Reprod 2013;28:3111-3117.

7. Van Roode T, Sharples K, Dickson N, et al. Life-course relationship between socioeconomic circumstances and timing of first birth in a birth cohort. PLoS One 2017;12: e0170170.

8. Abdou CM. Age-based reproductive healthcare stereotype threat (HCST) as a stressor affecting prenatal mental health in pregnant women of advanced maternal age: Measurement, process, outcomes, and interactions with ethnicity/race, SES, and other social identities. Curr Epidemiol Rep 2017;4:133-144.

9. Fretts RC, Usher RH. Causes of fetal death in women of advanced maternal age. Obstet Gynecol 1997;89:40-45.
10. van Katwijk C, Peeters LL. Clinical aspects of pregnancy after the age of 35 years: A review of the literature. Hum Reprod Update 1998;4:185-194.

11. Lee S, Holden D, Webb R, et al. Pregnancy related risk perception in pregnant women, midwives and doctors: A cross-sectional survey. BMC Pregnancy Childbirth 2019; 19:335.

12. Evans EC. A review of cultural influence on maternal mortality in the developing world. Midwifery 2013;29:490-496.

13. Ndiaye K, Portillo E, Ouedraogo D, et al. High-risk advanced maternal age and high parity pregnancy: Tackling a neglected need through formative research and action. Glob Health Sci Pract 2018;6:372-383.

14. Bayrampour H, Heaman M, Duncan KA, et al. Advanced maternal age, and risk perception: A qualitative study. BMC Pregnancy Childbirth 2012;12:100.

15. Tyer-Viola LA, Lopez RP. Pregnancy with chronic illness. JOGNN 2014;43:25-37.

16. Dulitzki M, Soriano D, Schiff E, et al. Effect of very advanced maternal age on pregnancy outcome and rate of cesarean delivery. Obstet Gynecol 1998;92:935-939.

17. Bianco A, Stone J, Lynch L, et al. Pregnancy outcome at age 40 and older. Obstet Gynecol 1996;87:917-922.

18. Jolly M, Sebire N, Harris J, et al. The risks associated with pregnancy in women aged 35 years or older. Hum Reprod 2000;15:2433-2437.

19. Cleary-Goldman J, Malone FD, Vidaver J, et al. Impact of maternal age on obstetric outcome. Obstet Gynecol 2005; 105:983-990.

20. Schummers L, Hutcheon JA, Hernandez-Diaz S, et al. Association of short interpregnancy interval with pregnancy outcomes according to maternal age. JAMA Intern Med 2018;178:1661-1670.

21. Sheen JJ, Wright JD, Goffman D, et al. Maternal age and risk for adverse outcomes. Am J Obstet Gynecol 2018;219: 390.e1.

22. Zeitlin JA, Ancel PY, Saurel-Cubizolles MJ, et al. Are risk factors the same for small for gestational age versus other preterm births? Am J Obstet Gynecol 2001;185: 208-215.

23. Paulson RJ, Boostanfar R, Saadat P, et al. Pregnancy in the sixth decade of life: Obstetric outcomes in women of advanced reproductive age. JAMA 2002;288:2320-2323.

24. Aliyu MH, Salihu HM, Wilson RE, et al. The risk of intrapartum stillbirth among smokers of advanced maternal age. Arch Gynecol Obstet 2008;278:39-45.

25. Fitzpatrick KE, Tuffnell D, Kurinczuk JJ, et al. Pregnancy at very advanced maternal age: A UK population-based cohort study. BJOG 2016;124:1097-1106.

26. Hoyert DL, Miniño AM. Maternal mortality in the United States: Changes in coding, publication, and data release, 2018. National vital statistics reports; vol 69, no 2. Hyattsville, MD: National Center for Health Statistics, 2020.

27. Shipp TD, Zelop C, Repke JT, et al. The association of maternal age and symptomatic uterine rupture during a trial of labor after prior cesarean delivery. Obstet Gynecol 2002; 99:585-588.

28. Callaghan WM, Berg CJ. Pregnancy-related mortality among women aged 35 years and older, United States, 1991-1997. Obstet Gynecol 2003;102:1015-1021.

29. Chang J, Elam-Evans LD, Berg CJ, et al. Pregnancy-related mortality surveillance-United States, 1991-1999. MMWR Surveill Summ 2003;52:1-8. 
30. Nybo Andersen AM, Wohlfahrt J, Christens P, et al. Maternal age and fetal loss: Population-based register linkage study. BMJ 2000;320:1708-1712.

31. Farr SL, Schieve LA, Jamieson DJ. Pregnancy loss among pregnancies conceived through assisted reproductive technology, United States, 1999-2002. Am J Epidemiol 2007; 165:1380-1388.

32. Sandin S, Schendel D, Magnusson P, et al. Autism risk associated with parental age and with increasing difference in age between the parents. Mol Psychiatry 2016;21: 693-700.

33. Liu L, Franco S, Spyropoulos B, et al. Irregular telomeres impair meiotic synapsis and recombination in mice. Proc Natl Acad Sci U S A 2004;101:6496-6501.

34. Hook EB. Rates of chromosome abnormalities at different maternal ages. Obstet Gynecol 1981;58:282-285.

35. Rowsey R, Gruhn J, Broman KW, Hunt PA, Hassold T. Examining variation in recombination levels in the human female: A test of the production-line hypothesis. Am J Hum Genet 2014;95:108-112.

36. Kolevzon A, Gross R, Reichenberg A. Prenatal and perinatal risk factors for autism: A review and integration of findings. Arch Pediatr Adolesc Med 2007;161:326-333.

37. Cohen D, Eisdorfer C, Leverenz J. Alzheimer's disease and maternal age. J Am Geriatr Soc 1982;30:656-659.

38. Rocca WA, Van Duijn CM, Clayton D, et al. Maternal age and Alzheimer's disease: A collaborative re-analysis of case-control studies. Int J Epidemiol 1991;20(Suppl_2): S21-S27.

39. Hassold T, Chiu D. Maternal age-specific rates of numerical chromosome abnormalities with special reference to trisomy. Hum Genet 1985;70:11-17.

40. Magnus MC, Wilcox AJ, Morken NH, Weinberg CR, Håberg SE. Role of maternal age and pregnancy history in risk of miscarriage: Prospective register-based study. BMJ 2019;364:1869.

41. Smith KE, Buyalos RP. The profound impact of patient age on pregnancy outcome after early detection of fetal cardiac activity. Fertil Steril 1996;65:35-40.

42. Spandorfer SD, Davis OK, Barmat LI, et al. Relationship between maternal age and aneuploidy in in vitro fertilization pregnancy loss. Fertil Steril 2004;81:1265-1269.

43. Miller DA. Is advanced maternal age an independent risk factor for uteroplacental insufficiency? Am J Obstet Gynecol 2005;192:1974-1980.

44. Burgess A, Founds S. Cardiovascular implications of preeclampsia. MCN Am J Matern Child Nurs 2016;41:8-15.

45. Wu J, Meldrum S, Dozier A, et al. Contraceptive nonuse among US women at risk for unplanned pregnancy. Contraception 2008;78:284-289.

46. Schmidt P, Skelly CL, Raines DA. Placental abruption (abruptio placentae). Treasure Island, FL: StatPearls Publishing, 2020.

47. Kaur K, Bhardwaj M, Kumar P, et al. Amniotic fluid embolism. J Anaesthesiol Clin Pharmacol 2016;32:153-159.

48. Materna-Kiryluk A, Wiśniewska K, Badura-Stronka M, et al. Parental age as a risk factor for isolated congenital malformations in a Polish population. Paediatr Perinat Epidemiol 2009;23:29-40.

49. Honigberg MC, Givertz MM. Peripartum cardiomyopathy. BMJ 2019;364:k5287.

50. Grobman WA, Rice MM, Reddy UM, et al. Labor induction versus expectant management in low-risk nulliparous women. N Engl J Med 2018;379:513-523.
51. Hedegaard M, Lidegaard $\varnothing$, Skovlund CW, et al. Reduction in stillbirths at term after new birth induction paradigm: Results of a national intervention. BMJ Open 2014;4:e005785.

52. Waldenström U, Cnattingius S, Vixner L, et al. Advanced maternal age increases the risk of very preterm birth, irrespective of parity: A population-based register study. BJOG 2017;124:1235-1244.

53. Richards MK, Flanagan MR, Littman AJ, et al. Primary cesarean section, and adverse delivery outcomes among women of very advanced maternal age. J Perinatol 2016;36:272-277.

54. Osterman MJ, Martin JA. Primary cesarean delivery rates, by state: Results from the revised birth certificate, 20062012. Natl Vital Stat Rep 2014;63:1-11.

55. Main DM, Main EK, Moore DH, 2nd. The relationship between maternal age and uterine dysfunction: A continuous effect throughout reproductive life. Am J Obstet Gynecol 2000;182:1312-1320.

56. Lin HC, Xirasagar S. Maternal age, and the likelihood of a maternal request for cesarean delivery: A 5-year population-based study. Am J Obstet Gynecol 2005;192: 848-855.

57. Treacy A, Robson M, O'Herlihy C. Dystocia increases with advancing maternal age. Am J Obstet Gynecol 2006;195: $760-763$

58. Greenberg MB, Cheng YW, Sullivan M, et al. Does length of labor vary by maternal age? Am J Obstet Gynecol 2007; 197:428.e1.

59. Zaki MN, Hibbard JU, Kominiarek MA. Contemporary labor patterns and maternal age. Obstet Gynecol 2013;122: 1018-1124.

60. Bujold E, Hammoud AO, Hendler I, et al. Trial of labor in patients with a previous cesarean section: Does maternal age influence the outcome? Am J Obstet Gynecol 2004; 190:1113-1118.

61. Walker KF, Malin G, Wilson P, et al. Induction of labour versus expectant management at term by subgroups of maternal age: An individual patient data meta-analysis. Eur J Obstet Gynecol Reprod Biol 2016;197:1-5.

62. Cohen W. Does maternal age affect pregnancy outcome? BJOG 2014;121:252-254.

63. Wang Y, Tanbo T, Abyholm T, et al. The impact of advanced maternal age and parity on obstetric and perinatal outcomes in singleton gestations. Arch Gynecol Obstet 2011;284:31-37.

64. Li Y, Townend J, Rowe R, et al. The effect of maternal age and planned place of birth on intrapartum outcomes in healthy women with straightforward pregnancies: Secondary analysis of the Birthplace National Prospective Cohort Study. BMJ Open 2014;4:e004026.

65. Pinheiro RL, Areia AL, Mota Pinto A, et al. Advanced maternal age: Adverse outcomes of pregnancy. A metaanalysis. Acta Med Port 2019;32:219-226.

66. Fall CH, Sachdev HS, Osmond C, et al. Association between maternal age at childbirth and child and adult outcomes in the offspring: A prospective study in five lowincome and middle-income countries (COHORTS collaboration). Lancet Glob Health 2015;3:e366-e377.

67. Casey BM, Lucas MJ, Mcintire DD, et al. Pregnancy outcomes in women with gestational diabetes compared with the general obstetric population. Obstet Gynecol 1997;90: 869-873.

68. Jacobsson B, Ladfors L, Milsom I. Advanced maternal age, and adverse perinatal outcome. Obstet Gynecol 2004;104: 727-733. 
69. Delpisheh A, Brabin L, Attia E, et al. Pregnancy late in life: A hospital-based study of birth outcomes. J Womens Health (Larchmt) 2008;17:965-970.

70. Cnattingius S, Forman MR, Berendes HW, et al. Delayed childbearing and risk of adverse perinatal outcome. A population-based study. JAMA 1992;268:886-890.

71. Goisis A, Remes H, Barclay K, et al. Advanced maternal age and the risk of low birth weight and preterm delivery: A within-family analysis using Finnish population registers. Am J Epidemiol 2017;186:1219-1226.

72. Reddy UM, Ko CW, Willinger M. Maternal age and the risk of stillbirth throughout pregnancy in the United States. Am J Obstet Gynecol 2006;195:764-770.

73. Flenady V, Koopmans L, Middleton P, et al. Major risk factors for stillbirth in high-income countries: A systematic review and meta-analysis. Lancet 2011;377:1331-1340.

74. Canterino JC, Ananth CV, Smulian J, et al. Maternal age and risk of fetal death in singleton gestations: USA, 19952000. J Matern Fetal Neonatal Med 2004;15:193-197.

75. Bateman BT, Simpson LL. Higher rate of stillbirth at the extremes of reproductive age: A large nationwide sample of deliveries in the United States. Am J Obstet Gynecol 2006; 194:840-845.

76. Haavaldsen C, Sarfraz AA, Samuelsen SO, et al. The impact of maternal age on fetal death: Does length of gestation matter? Am J Obstet Gynecol 2010;203:554.e1.

77. Lisonkova S, Potts J, Muraca GM, et al. Maternal age and severe maternal morbidity: A population-based retrospective cohort study. PLoS Med 2017;14:e1002307.

78. Lao TT, Sahota DD, Cheng YK, et al. Advanced maternal age and postpartum hemorrhage-Risk factor or red herring? J Matern Fetal Neonatal Med 2014;27:243-246.

79. Qureshi AI, Saeed O, Malik AA, et al. Pregnancy in advanced age and the risk of stroke in postmenopausal women: Analysis of Women's Health Initiative Study. Am J Obstet Gynecol 2017;216:409.E1-409.E6.

80. Mosca L, Benjamin EJ, Berra K, et al. Effectiveness-based guidelines for the prevention of cardiovascular disease in women-2011 update: A guideline from the American Heart Association. J Am Coll Cardiol 2011;57:1404-1423.

81. Jaffe D, Kogan L, Manor O, et al. Influence of late-age births on maternal longevity. Ann Epidemiol 2015;25:387391.

82. Muraca GM, Joseph KS. The association between maternal age and depression. J Obstet Gynaecol Can 2014;36:803810.

83. Mielke MM, Milic NM, Weissgerber TL, et al. Impaired cognition and brain atrophy decades after hypertensive pregnancy disorders. Circ Cardiovasc Qual Outcomes 2016;9(Suppl 1):S70-S76.

84. Nebel RA, Aggarwal NT, Barnes LL, et al. Understanding the impact of sex and gender in Alzheimer's disease: A call to action. Alzheimers Dement 2018;14:1171-1183.

85. Sun F, Sebastiani P, Schupf N, et al. Extended maternal age at birth of last child and women's longevity in the LongLife Family Study. Menopause 2015;22:26-31.

Address correspondence to: Rosaly Correa-de-Araujo, MD, MSc, PhD Division of Geriatrics and Clinical Gerontology U.S. Department of Health and Human Services National Institute on Aging National Institutes of Health 7201 Wisconsin Avenue, Suite 3W200 Bethesda, MD 20892

USA

E-mail: rosaly.correa-de-araujo@nih.gov 